\title{
Exploiting trial-to-trial variability in multimodal experiments
}

\author{
Amy R. Bland ${ }^{1}$, Faisal Mushtaq ${ }^{1}$ and David V. Smith ${ }^{2,3}$ \\ 1 Institute of Psychological Sciences, University of Leeds, Leeds, UK \\ 2 Center for Cognitive Neuroscience, Duke University, Durham, NC, USA \\ ${ }^{3}$ Department of Psychology and Neuroscience, Duke University, Durham, NC, USA \\ *Correspondence: a.r.bland04@leeds.ac.uk
}

\section{A commentary on}

Effects of parametrical and trial-to-trial variation in prior probability processing revealed by simultaneous electroencephalogram/functional magnetic resonance imaging

by Scheibe, C., Ullsperger, M., Sommer, W., and Heekeren, H. R. (2010). J. Neurosci. 30, 16709-16717.

Event-related potentials (ERP) observed in the electroencephalogram (EEG) have traditionally provided neural markers for an array of cognitive phenomena through averaging time-locked amplitudes over many trials. However, it is becoming clear that understanding trial-to-trial variability in neural activity and its behavioral consequences is an important venture in cognitive and systems neuroscience. Recent studies have begun to focus on how fluctuations in functional magnetic resonance imaging (fMRI) and electrophysiological (EEG/MEG) signals are correlated with moment-to-moment fluctuations in behavior (e.g., Fox et al., 2005; Pessoa and Padmala, 2005; Mars et al., 2008). Indeed, neural responses can vary in theoretically important ways which may reflect a signature of task-relevant brain-state changes such as a subject's cognitive "context" (Lutz et al., 2002). As such, focusing on single-trial data can provide a more direct link between neural activity and cognitive processes, such as executive function and decision making (Debener et al., 2006).

Examining trial-to-trial variability may provide a unique window for exploring dynamic modulations in the decisionmaking process, which frequently requires computing many variables that inform and optimize the decision (e.g., Mulert et al., 2005; Weissman et al., 2006; Esposito et al., 2009). One such variable is prior probability (PP), the prior knowledge of the probability concerning the decision alternatives. This PP must be integrated into the decision-making process to form expectations about which event is the most probable to occur. Behavioral studies have previously shown that PP modulates behavior, with increasing PP predicting faster reaction times (e.g., Carpenter, 2004). Neural correlates of this behavioral effect have been linked to changes in the contingent negative variation $(\mathrm{CNV})$, whose mean amplitude increases with increasing PP (Scheibe et al., 2009). Nevertheless, while mean amplitude ERPs such as the CNV can provide insight into PP processing, it neglects to consider trialspecific neural processes and their associated brain regions.

Scheibe et al. (2010) tackled this problem in a recent issue of The Journal of Neuroscience by simultaneously recording EEG and fMRI as participants performed a simple number comparison task (Scheibe et al., 2010; Figure 1A). On each trial, participants saw a fixation cross, followed by a number $(S 1 ; 1,3,5,7,9)$ presented at either side. After 2000 ms, a second number (S2; any number not used in S1) was then displayed at the opposite side of the fixation and remained until the participant responded by indicating the numerically larger number. Essentially, S1 served as a cue with information about the probability of the side with the largest value with a predictive value of 1.0 ( 1 or 9$), 0.75$ (3 or 5), or 0.5 (5). As expected, this design produced a robust parametric behavioral effect of PP, with increasing PP predicting faster RTs (Scheibe et al., 2010; Figure 1B).

When examining the neural basis of these behavioral effects, Scheibe et al. (2010) observed substantial trial-to-trial variability in the CNV response (Scheibe et al., 2010; Figure 2A). To identify the neural correlates of trial-to-trial fluctuations of PP processing, Scheibe et al. (2010) conducted three distinct analyses. First, the authors identified voxels parametrically modulated by PP. Using this method, they demonstrated that the posterior medial frontal cortex (pMFC) increased parametrically with increasing PP. Next, Scheibe et al. (2010) employed an EEG-informed fMRI analysis, which utilizes the variability in EEG data to predict changes in BOLD response (e.g., Goldman et al., 2009). In order to identify voxels whose activity covaried with the CNV, Scheibe et al. (2010) added a regressor using the CNV amplitude for each trial. This identified a network of regions including the middle frontal gyrus (MFG), dorsolateral prefrontal cortex (DLPFC), and putamen (Scheibe et al., 2010; Figure 4A). The authors then regressed out the variance attributable to PP, and used the residuals in a follow up regression model. Finally, by comparing the model derived from the EEG-informed fMRI analysis and the follow up regression model, regions that were only activated in the former and not the latter model were identified as being attributable only to PP processing. Here, the authors showed that the DLPFC, right inferior frontal gyrus (IFG), and right inferior parietal lobule (IPL; Scheibe et al., 2010; Figure 4B) were modulated exclusively by the processing and integration of PP into response preparation.

These results provide novel insights into trial-specific information processing of PP during decision making. Scheibe et al. (2010) show that the neural integration of PP has at least three levels. Firstly, increasing PP indexes the need for behavioral adjustment, which is reflected by increases in pMFC and the CNV in order to successfully prepare motor responses and guide future decisions. Previously, the pMFC has been shown to be involved in performance monitoring and cognitive control induced behavioral adjustments (Ridderinkhof et al., 2004). Next, Scheibe et al. (2010) were able to identify regions that covaried with the $\mathrm{CNV}$, which they suggest might index unspecific 
preparatory processes. Finally, regions including the DLPFC were exclusively related to $\mathrm{PP}$-induced $\mathrm{CNV}$ fluctuations. Given the role of the DLPFC in establishing, regulating, and actively maintaining attention (MacDonald et al., 2000; Weissman et al., 2006), the authors suggest that this reflects trial-specific attentional effort, depending on the probability information. However, it is worth noting that the exact cognitive mechanisms would need to be elucidated with future studies that manipulate attentional control.

In addition, the results of Scheibe et al. (2010) are accompanied by at least two caveats. First, methods that alter the relationship between correlated factors in a general linear model (e.g., orthogonalization) can sometimes impose interpretational challenges on the resulting estimates, depending on the degree of correlation between the factors (Hunt, 2008). Applying this logic to Scheibe et al. (2010) it is important to note that their analyses proceeded in three stages, with the third and final stage utilizing the residuals from a previous - but related - model. Although this approach allows Scheibe et al. (2010) to partial out variance that is attributable to PP, the results and interpretations could depend on the correlation between the original model factors. Second, while Scheibe et al. (2010) used a 200-ms time window for their mean amplitude CNV analysis, they used a significantly larger window of $1000 \mathrm{~ms}$ in their single-trial analysis of the CNV. This $1000 \mathrm{~ms}$ epoch could potentially conflate other processes with the CNV quantification. Indeed, the $\mathrm{CNV}$ may have at least two phases: an early, frontal phase relating to orientation and a later, more posterior phase relating to the motor preparation of the response (Gómez et al., 2003). By focusing their analysis on a large temporal window over one central electrode site (Cz), Scheibe et al. (2010) may have failed to capture distinct processes relating the $\mathrm{CNV}$.

More generally, concurrent EEG-fMRI recordings also present some key challenges for researchers. Importantly, either method alone suffers from a lack of consistency in distinguishing between trial-to-trial variability that is functionally significant and variability that is simply noise. There are many algorithms that have been proposed for quantifying trial-to-trial variability in EEG data. These include techniques such as hierarchical Bayesian modeling (Wu et al., 2011), linear dynamical system response and independent response modeling (Limpiti et al., 2009). Nevertheless, estimating trialto-trial variability still represents an important venture in future research in order to adequately utilize this in EEG-informed fMRI analysis. Particularly important to multimodal studies is that fMRI does not necessarily identify the neural generators of EEG signals as each can differ in their sensitivity to experimental manipulations (Debener et al., 2006). Indeed, the variability observed in the electrophysiological data may not necessarily be reflected in the fMRI signal. Vartiainen et al. (2011) recently tested this issue empirically, demonstrating that hemodynamic and electrophysiological measures show strikingly different functional patterns within the same tasks. Exploring these differences should serve as a first step toward a principled combined use of hemodynamic and electrophysiological measures.

Despite these challenges, Scheibe et al. (2010) provide novel insights into the neural mechanisms of PP processing - thus highlighting the power of multimodal studies. However, it could also be important probe the dynamic interplay between intrinsic and task-evoked brain activity (Northoff et al., 2010). Indeed, neuroscience has only begun to fully exploit the dynamic changes in restingstate networks and link those to changes in mental function. The brain can be considered a dynamic system which can itself determine properties of the response to any given stimulus (Debener et al., 2006) and so intrinsic ongoing brain activity may also be an important determinant of the trial-to-trial fluctuations observed in Scheibe et al. (2010). Along these lines, behavioral performance should not automatically be attributed to fluctuations in task-related cognitive processes, as ongoing fluctuations in intrinsic neuronal activity have been shown to play an important role (Fox et al., 2007). Elucidating the relationship between these two forms of brain activity could provide a unique window for examining the cause of trial-to-trial variability in brain and behavior.

\section{ACKNOWLEDGMENTS}

We wish to thank Dr. Scott Huettel and Rene San Martin for helpful comments and discussion regarding this commentary. We were supported by an ESRC grant (Amy R. Bland) and by a NIMH National Research Service Award F31-086248 (David Victor Smith).

\section{REFERENCES}

Carpenter, R. H.S. (2004). Contrast, probability, and saccadic latency: evidence for independence of detection and decision. Curr. Biol. 14, 1576-1580.

Debener, S., Ullsperger, M., Siegel, M., and Engel, A. K. (2006). Single-trial EEG-fMRI reveals the dynamics of cognitive function. Trends Cogn. Sci. (Regul. Ed.) $10,558-563$.

Esposito, F., Mulert, C., and Goebel, R. (2009). Combined distributed source and single-trial EEG-fMRI modeling: application to effortful decision making processes. Neuroimage 47, 112-121.

Fox, M. D., Snyder, A. Z., Vincent, J. L., Corbetta, M., Van Essen, D. C., and Raichle, M. E. (2005). The human brain is intrinsically organized into dynamic, anticorrelated functional networks. Proc. Natl. Acad. Sci. U.S.A. 102, 9673-9678.

Fox, M. D., Snyder, A. Z., Vincent, J. L., and Raichle, M. E. (2007). Intrinsic fluctuations within cortical systems account for intertrial variability in human behavior. Neuron 56, 171-184.

Goldman, R. I., Wei, C.-Y., Philiastides, M. G., Gerson, A. D., Friedman, D., Brown, T. R., and Sajda, P. (2009) Single-trial discrimination for integrating simultaneous EEG and fMRI: identifying cortical areas contributing to trial-to-trial variability in the auditory oddball task. Neuroimage 47, 136-147.

Gómez, C. M., Marco, J., and Grau, C. (2003). Preparatory visuo-motor cortical network of the contingent negative variation estimated by current density. Neuroimage 20, 216-224.

Hunt, L. (2008). Distinctive roles for the ventral striatum and ventral prefrontal cortex during decision-making. J. Neurosci. 28, 8658-8659.

Limpiti, T., Van Veen, B. D., Attias, H. T., and Nagarajan, S. S. (2009). A spatiotemporal framework for estimating trial-to-trial amplitude variation in eventrelated MEG/EEG. IEEE Trans. Biomed. Eng. 56, 633-645.

Lutz, A., Lachaux, J.-P., Martinerie, J., and Varela, F. J. (2002). Guiding the study of brain dynamics by using first-person data: synchrony patterns correlate with ongoing conscious states during a simple visual task. Proc. Natl. Acad. Sci. U.S.A. 99, 1586-1591.

MacDonald, A. W., Cohen, J. D., Stenger, V.A., and Carter, C.S. (2000). Dissociating the role of the dorsolateral prefrontal and anterior cingulate cortex in cognitive control. Science 288, 1835-1838.

Mars, R. B., Debener, S., Gladwin, T. E., Harrison, L. M., Haggard, P., Rothwell, J. C., and Bestmann, S. (2008). Trial-by-trial fluctuations in the eventrelated electroencephalogram reflect dynamic changes in the degree of surprise. J. Neurosci. 28, 12539-12545.

Mulert, C., Menzinger, E., Leicht, G., Pogarell, O., and Hegerl, U. (2005). Evidence for a close relationship 
between conscious effort and anterior cingulate cortex activity. Int. J. Psychophysiol. 56, 65-80.

Northoff, G., Qin, P., and Nakao, T. (2010). Rest-stimulus interaction in the brain: a review. Trends Neurosci. 33, 277-284.

Pessoa, L., and Padmala, S. (2005). Quantitative prediction of perceptual decisions during near-threshold fear detection. Proc. Natl. Acad. Sci. U.S.A. 102, 5612-5617.

Ridderinkhof, K. R., van den Wildenberg, W. P. M., Segalowitz, S. J., and Carter, C. S. (2004). Neurocognitive mechanisms of cognitive control: the role of prefrontal cortex in action selection, response inhibition, performance monitoring, and rewardbased learning. Brain Cogn. 56, 129-140.

Scheibe, C., Schubert, R., Sommer, W., and Heekeren, H. R. (2009). Electrophysiological evidence for the effect of prior probability on response preparation. Psychophysiology 46, 758-770.

Scheibe, C., Ullsperger, M., Sommer, W., and Heekeren, H. R. (2010). Effects of parametrical and trialto-trial variation in prior probability processing revealed by simultaneous electroencephalogram/ functional magnetic resonance imaging. J. Neurosci. 30, 16709-16717.

Vartiainen, J., Liljestrom, M., Koskinen, M., Renvall, H., and Salmelin, R. (2011). Functional magnetic resonance imaging blood oxygenation level-dependent signal and magnetoencephalography evoked responses yield different neural functionality in reading. J. Neurosci. 31, 1048-1058.

Weissman, D. H., Roberts, K. C., Visscher, K. M., and Woldorff, M. G. (2006). The neural bases of momentary lapses in attention. Nat. Neurosci. 9, 971-978.
Wu, W., Chen, Z., Gao, S., and Brown, E. N. (2011). A hierarchical Bayesian approach for learning sparse spatio-temporal decompositions of multichannel EEG. Neuroimage 56, 1929-1945.

Received: 28 February 2011; accepted: 26 July 2011; published online: 15 August 2011.

Citation: Bland AR, Mushtaq F and Smith DV (2011) Exploiting trial-to-trial variability in multimodal experiments. Front. Hum. Neurosci. 5:80. doi: 10.3389/ fnhum.2011.00080

Copyright (C) 2011 Bland, Mushtaq and Smith. This is an open-access article subject to a non-exclusive license between the authors and Frontiers Media SA, which permits use, distribution and reproduction in other forums, provided the original authors and source are credited and other Frontiers conditions are complied with. 